\title{
Article
}

\section{Existence of solution a fractional differential equation}

\section{Zouaoui Bekri ${ }^{1, *}$ and Slimane Benaicha ${ }^{2}$}

1 Laboratory of fundamental and applied mathematics, University of Oran 1, Ahmed Ben Bella, Es-senia, 31000 Oran, Algeria.

2 Laboratory of fundamental and applied mathematics, University of Oran 1, Ahmed Ben Bella, Es-senia, 31000 Oran, Algeria.

* Correspondence: zouaouibekri@yahoo.fr

Received: 29 June 2020; Accepted: 24 September 2020; Published: 7 October 2020.

Abstract: In this paper, we study the existence of nontrivial solution for the fractional differential equation of order $\alpha$ with three point boundary conditions having the following form

$$
\begin{gathered}
D^{\alpha} u(t)=f\left(t, v(t), D^{v} v(t)\right), \quad t \in(0, T) \\
u(0)=0, \quad u(T)=a u(\xi),
\end{gathered}
$$

where $1<\alpha<2, v, a>0, \xi \in(0, T), T^{\alpha-1}+a \xi^{\alpha-1} \neq 0 . D$ is the standard Riemann-Liouville fractional derivative operator and $f \in C\left([0,1] \times \mathbf{R}^{2}, \mathbf{R}\right)$. Applying the Leray-Schauder nonlinear alternative we prove the existence of at least one solution. As an application, we also given some examples to illustrate the results obtained.

Keywords: Caputo derivative of fractional order, Leary-Schauder nonlinear alternative, fixed point theorem, Riemann-Liouville fractional integral, fractional differential equations.

MSC: 34A08, 34A34, 36A33.

\section{Introduction}

$\mathbf{F}$ ractional differential equations arise in many engineering and scientific disciplines as the mathematical modeling of systems and processes in the fields of physics, chemistry, aerodynamics, electrodynamics of complex medium, polymer rheology, Bode's analysis of feedback amplifiers, signal and image processing, capacitor theory, electrical circuits, electron-analytical chemistry, biology, ow in porous media, aerodynamics, viscoelasticity, control theory, fitting of experimental data, and so forth, and involves derivatives of fractional order. Fractional derivatives provide an excellent tool for the description of memory and hereditary properties of various materials and processes (for details, see [1-9]). The fractional differential equations under various conditions have been studied by ([10-13]), etc. The three point boundary value problem given by a coupled system of FDE on the interval $(0,1)$ was studied by Bashir [10]

$$
\begin{cases}D^{\alpha} u(t)=f\left(t, v(t), D^{p_{v}}(t)\right), & t \in(0,1) \\ D^{\beta} v(t)=f\left(t, u(t), D^{q} u(t)\right), & t \in(0,1) \\ u(0)=0, u(1)=a u(\xi), v(0)=0, v(1)=a v(\xi), & \end{cases}
$$

where $1<\alpha, \beta<2, p, q, a>0,0<\xi<1, \alpha-q \geq 1, \beta-p \geq 1, a \xi^{\alpha-1}<1$ and $a \xi^{\beta-1}<1$. $D$ is the standard Riemann-Liouville fractional derivative operator and $f:[0,1] \times \mathbf{R}^{2} \longrightarrow \mathbf{R}^{2}$.

Infinite systems of ODE's was first studied by Persidskii [14] with the aid of classical tools such as successive approximation and the classical Banach fixed point principle. The infinite systems of differential equations emerge in study of various topics of nonlinear analysis. For example semidiscretization of certain parabolic partial differential equation leads to an infinite system of ODE [15], while modeling certain physical phenomenon in theory of neural sets, branching process and mechanics $([16,17])$, where the infinite system 
can be represented as an ordinary differential equation. Consider the following infinite system of fractional differential equations [18]

$$
\begin{cases}D^{\alpha} u_{i}(t)=f_{i}(t, u(t)), & t \in(0, T) \\ u_{i}(0)=u_{i}^{0}=0, \quad u_{i}(T)=a u_{i}(\xi), & i=1,2,3 \ldots \\ 1<\alpha<2, \quad a \xi^{\alpha-1}<T^{\alpha-1} & \end{cases}
$$

where each $u_{i}(t)$ is a differentiable function of class $C^{[\alpha]+1}$. We will denote the sequence $\left\{u_{i}(t)\right\}_{i=1}^{\infty}=u(t)$, $\left\{u_{i}(0)\right\}_{i=1}^{\infty}=u_{0},\left\{u_{i}(\xi)\right\}_{i=1}^{\infty}=u(\xi)$ and $\left\{f_{i}(t, u(t))\right\}_{i=1}^{\infty}=f(t, u(t))$ which is an element of some Banach sequence space $(E,\|\cdot\|)$.

Motivated by the above works, the aim of this paper is to establish some sufficient conditions for the existence of nontrivial solution for the fractional differential equations (FDE) as follows

$$
\left\{\begin{array}{l}
D^{\alpha} u(t)=f\left(t, v(t), D^{v} v(t)\right), \quad t \in(0, T) \\
u(0)=0, \quad u(T)=a u(\xi),
\end{array}\right.
$$

where $1<\alpha<2 ; v, a>0, \xi \in(0, T) ; \alpha-\mu \geq 1$ and $T^{\alpha-1}+a \xi^{\alpha-1} \neq 0$. D is the standard Riemann-Liouville fractional derivative operator and $f \in C\left([0,1] \times \mathbf{R}^{2}, \mathbf{R}\right)$.

This paper is organized as follows. In Section 2, we present some definitions and lemmas that will be used to prove the results. Then, in Section 3, we present and prove our main results which consists of existence theorems and corollary for nontrivial solution of the FDE 3, and we establish some existence criteria of at least one solution by using the Leray-Schauder nonlinear alternative. Finally, in Section 4, as an application, we give some examples to illustrate the results we obtained.

\section{Preliminaries}

In this section, we introduce some necessary definitions and lemmas of fractional calculus to facilitate the analysis of the Problem (3). These details can be found in the recent literature, see ([3,7,19-23]) and the references therein.

Definition 1. Let $\alpha>0, n-1<\alpha<n, n=[\alpha]+1$ and $u \in C([0,1), \mathbf{R})$. The Caputo derivative of fractional order $\alpha$ for the function $u$ is defined by

$$
{ }^{c} D^{\alpha} u(t)=\frac{1}{\Gamma(n-\alpha)} \int_{0}^{t}(t-s)^{n-\alpha-1} u^{(n)}(s) d s,
$$

where $\Gamma(\cdot)$ is the Gamma function.

Definition 2. The Riemann-Liouville fractional integral of order $\alpha>0$ of a function $u:(0, \infty) \longrightarrow \mathbf{R}$ is given by

$$
I^{\alpha} u(t)=\frac{1}{\Gamma(\alpha)} \int_{0}^{t}(t-s)^{\alpha-1} u(s) d s, t>0,
$$

where $\Gamma(\cdot)$ is the Gamma function, provided that the right side is pointwise defined on $(0, \infty)$.

Lemma 1. ([23]) Let $\alpha, \beta>0$ and $u \in L^{p}(0,1), 1 \leq p \leq+\infty$. Then the next formulas hold;

(i) $\left(I^{\beta} I^{\alpha} u\right)(t)=I^{\alpha+\beta} u(t)$,

(ii) $\left(D^{\beta} I^{\alpha} u\right)(t)=I^{\alpha-\beta} u(t)$,

(iii) $\left(D^{\alpha} I^{\alpha} u\right)(t)=u(t)$.

Lemma 2. Let $\alpha>0, n-1<\alpha<n$ and the function $g:[0 ; T] \longrightarrow \mathbf{R}$ be continuous for each $T>0$. Then, the general solution of the fractional differential equation ${ }^{c} D^{\alpha} g(t)=0$ is given by

$$
g(t)=c_{0}+c_{1} t+\ldots+c_{n-1} t^{n-1},
$$

where $c_{0}, c_{1}, \ldots, c_{n-1}$ are real constants and $n=[\alpha]+1$. 
Lemma 3. Assume that $u \in C[0,1] \cap L^{1}(0,1)$ with a Caputo fractional derivative of order $\alpha>0$ that belongs to $u \in C^{n}[0,1]$, then

$$
I^{\alpha}{ }^{c} D^{\alpha} u(t)=u(t)+c_{0}+c_{1} t+\ldots+c_{n-1} t^{n-1},
$$

where $c_{0}, c_{1}, \ldots, c_{n-1}$ are real constants and $n=[\alpha]+1$.

Lemma 4. For $\alpha>0$, the general solution of the fractional differential equation $D^{\alpha} u(t)=0$ with $u \in C[0,1] \cap L^{1}(0,1)$ is given by

$$
u(t)=c_{1} t^{\alpha-1}+c_{2} t^{\alpha-2}+\ldots+c_{n} t^{\alpha-n}
$$

where $c_{i} \in \mathbf{R}, \quad i=1,2, \ldots, n$. Hence for $u \in C[0,1] \cap L^{1}(0,1)$, we have

$$
I^{\alpha} D^{\alpha} u(t)=u(t)+c_{1} t^{\alpha-1}+c_{2} t^{\alpha-2}+\ldots+c_{n} t^{\alpha-n}
$$

Lemma 5. Let $y \in C([0, T]), T^{\alpha-1}+a \xi^{\alpha-1} \neq 0$. Then FDE

$$
\left\{\begin{array}{l}
D^{\alpha} u(t)=y(t), \quad t \in(0, T) \\
u(0)=0, u(T)=a u(\xi)
\end{array}\right.
$$

has a unique solution

$$
\begin{aligned}
u(t)= & \frac{1}{\Gamma(\alpha)} \int_{0}^{t}\left[(t-s)^{\alpha-1}-\frac{(t(T-s))^{\alpha-1}}{\left(T^{\alpha-1}-a \xi^{\alpha-1}\right)}\right] y(s) d s-\frac{1}{\left(T^{\alpha-1}-a \xi^{\alpha-1}\right) \Gamma(\alpha)} \int_{t}^{T}(t(T-s))^{\alpha-1} y(s) d s \\
& +\frac{a}{\left(T^{\alpha-1}-a \xi^{\alpha-1}\right) \Gamma(\alpha)} \int_{0}^{\xi}(t(\xi-s))^{\alpha-1} y(s) d s
\end{aligned}
$$

Proof. ([10])The general solution of FDE is

$$
u(t)=I^{\alpha} y(t)+c_{1} t^{\alpha-1}+c_{2} t^{\alpha-2}, \text { where } c_{1}, c_{2} \in \mathbf{R}
$$

Using the boundary conditions, we find that $c_{2}=0$ and

$$
c_{1}=-\frac{1}{\left(T^{\alpha-1}-a \xi^{\alpha-1}\right)}\left[\int_{0}^{T} \frac{y(s) d s}{(T-s)^{\alpha-1} \Gamma(\alpha)}-a \int_{0}^{\xi} \frac{y(s) d s}{(\xi-s)^{\alpha-1} \Gamma(\alpha)}\right] .
$$

Substituting $c_{1}$ and $c_{2}$ by their values in $u(t)$, we obtain the solution in the statement of the lemma. This completes the proof.

Define the integral operator $F: E \rightarrow E$, by

$$
\begin{aligned}
F u(t)= & \frac{1}{\Gamma(\alpha)} \int_{0}^{t}\left[(t-s)^{\alpha-1}-\frac{(t(T-s))^{\alpha-1}}{\left(T^{\alpha-1}-a \xi^{\alpha-1}\right)}\right] f\left(s, v(s), D^{v} v(s)\right) d s \\
& -\frac{1}{\left(T^{\alpha-1}-a \xi^{\alpha-1}\right) \Gamma(\alpha)} \int_{t}^{T}(t(T-s))^{\alpha-1} f\left(s, v(s), D^{v} v(s)\right) d s \\
& +\frac{a}{\left(T^{\alpha-1}-a \xi^{\alpha-1}\right) \Gamma(\alpha)} \int_{0}^{\xi}(t(\xi-s))^{\alpha-1} f\left(s, v(s), D^{v} v(s)\right) d s .
\end{aligned}
$$

By Lemma 5, the FDE (3) has a solution if and only if the operator $F$ has a fixed point in $E$. So we only need to seek a fixed point of $F$ in $E$. By Ascoli-Arzela theorem, we can prove that $F$ is a completely continuous operator. Now we cite the Leray-Schauder nonlinear alternative.

Lemma 6. Let $E$ be a Banach space and $\Omega$ be a bounded open subset of $E, 0 \in \Omega$.F $: \bar{\Omega} \rightarrow$ E be a completely continuous operator. Then, either

(i) there exists $u \in \partial \Omega$ and $\lambda>1$ such that $F(u)=\lambda u$, or

(ii) there exists a fixed point $u^{*} \in \bar{\Omega}$ of $F$. 


\section{Main results}

In this section, we prove the existence of a nontrivial solution for the FDE (3). Let $E=C([0, T])$ with the norm $\|v\|=\max _{t \in[0, T]}\left\{|v(t)|,\left|D^{v} v(t)\right|\right\}$ for any $v \in E, f \in C\left([0, T] \times \mathbf{R}^{2}, \mathbf{R}\right)$.

Theorem 1. Suppose that $f(t, 0,0) \neq 0, T^{\alpha-1}+a \xi^{\alpha-1} \neq 0$, and there exist nonnegative functions $k, h, l \in L^{1}[0, T]$ such that

$$
|f(t, x, y)| \leq k(t)|x|+h(t)|y|+l(t), \quad \text { a.e. }(t, x, y) \in[0, T] \times \mathbf{R}^{2},
$$

and

$$
\frac{2 T^{\alpha-1}+a \xi^{\alpha-1}}{\left(T^{\alpha-1}+a \xi^{\alpha-1}\right) \Gamma(\alpha)} \int_{0}^{T}(T-s)^{\alpha-1}(k(s)+h(s)) d s+\frac{a T^{\alpha-1}}{\left(T^{\alpha-1}+a \xi^{\alpha-1}\right) \Gamma(\alpha)} \int_{0}^{\tau}(\xi-s)^{\alpha-1}(k(s)+h(s)) d s<1 .
$$

Then the FDE (3) has at least one nontrivial solution $u^{*} \in C([0, T])$.

Proof. Let

$$
A=\frac{2 T^{\alpha-1}+a \xi^{\alpha-1}}{\left(T^{\alpha-1}+a \xi^{\alpha-1}\right) \Gamma(\alpha)} \int_{0}^{T}(T-s)^{\alpha-1}(k(s)+h(s)) d s+\frac{a T^{\alpha-1}}{\left(T^{\alpha-1}+a \xi^{\alpha-1}\right) \Gamma(\alpha)} \int_{0}^{\xi}(\xi-s)^{\alpha-1}(k(s)+h(s)) d s,
$$

and

$$
B=\frac{2 T^{\alpha-1}+a \xi^{\alpha-1}}{\left(T^{\alpha-1}+a \xi^{\alpha-1}\right) \Gamma(\alpha)} \int_{0}^{T}(T-s)^{\alpha-1} l(s) d s+\frac{a T^{\alpha-1}}{\left(T^{\alpha-1}+a \xi^{\alpha-1}\right) \Gamma(\alpha)} \int_{0}^{\xi}(\xi-s)^{\alpha-1} l(s) d s,
$$

then $A<1$. Since $f(t, 0,0) \neq 0$, there exists an interval $[a, b] \subset[0,1]$ such that $\min _{a \leq t \leq b}|f(t, 0,0)|>0$, and, as $l(t) \geq|f(t, 0,0)|$, a.e., and $t \in[0, T]$, so $B>0$.

Let $C=B(1-A)^{-1}$ and $\Omega=\left\{(u, v) \in E^{2}:\|(u, v)\|_{E^{2}}<C\right\}$. Assume that $u \in \partial \Omega$ and $\lambda>1$ such that $F u=\lambda u$, then

$$
\begin{aligned}
\lambda C= & \lambda\|u\|=\|F u\|=\max _{0 \leq t \leq T}|(F u)(t)| \\
\leq & \frac{1}{\Gamma(\alpha)} \max _{t \in[0, T]} \int_{0}^{t}\left|(t-s)^{\alpha-1}-\frac{(t(T-s))^{\alpha-1}}{\left(T^{\alpha-1}-a \xi^{\alpha-1}\right)}\right| f\left(s, v(s), D^{v} v(s)\right) \mid d s \\
& +\max _{t \in[0, T]} \frac{1}{\left|T^{\alpha-1}-a \xi^{\alpha-1}\right| \Gamma(\alpha)} \int_{t}^{T}(t(T-s))^{\alpha-1}\left|f\left(s, v(s), D^{v} v(s)\right)\right| d s \\
& +\max _{t \in[0, T]} \frac{a}{\left|T^{\alpha-1}-a \xi^{\alpha-1}\right| \Gamma(\alpha)} \int_{0}^{\xi}(t(\xi-s))^{\alpha-1}\left|f\left(s, v(s), D^{v} v(s)\right)\right| d s \\
\leq & \frac{1}{\Gamma(\alpha)} \int_{0}^{T}\left[(T-s)^{\alpha-1}+\frac{(T(T-s))^{\alpha-1}}{\left(T^{\alpha-1}+a \xi^{\alpha-1}\right)}\right]\left|f\left(s, v(s), D^{v} v(s)\right)\right| d s \\
& +\frac{a}{\left(T^{\alpha-1}+a \xi^{\alpha-1}\right) \Gamma(\alpha)} \int_{0}^{\xi}(T(\xi-s))^{\alpha-1}\left|f\left(s, v(s), D^{v} v(s)\right)\right| d s \\
\leq & \frac{\left(2 T^{\alpha-1}+a \xi^{\alpha-1}\right)}{\left(T^{\alpha-1}+a \xi^{\alpha-1}\right) \Gamma(\alpha)} \int_{0}^{T}(T-s)^{\alpha-1}\left(k(s)|v(s)|+h(s)\left|D^{v} v(s)\right|+l(s)\right) d s \\
& +\frac{a T^{\alpha-1}}{\left(T^{\alpha-1}+a \xi^{\alpha-1}\right) \Gamma(\alpha)} \int_{0}^{\xi}(\xi-s)^{\alpha-1}\left(k(s)|v(s)|+h(s)\left|D^{v} v(s)\right|+l(s)\right) d s \\
\leq & {\left[\frac{\left(2 T^{\alpha-1}+a \xi^{\alpha-1}\right)}{\left(T^{\alpha-1}+a \xi^{\alpha-1}\right) \Gamma(\alpha)} \int_{0}^{T}(T-s)^{\alpha-1}(k(s)+h(s))\|v\| d s\right.} \\
& \left.+\frac{a T^{\alpha-1}}{\left(T^{\alpha-1}+a \xi^{\alpha-1}\right) \Gamma(\alpha)} \int_{0}^{\xi}(\xi-s)^{\alpha-1}(k(s)+h(s))\|v\| d s\right] \\
& +\left[\frac{\left(2 T^{\alpha-1}+a \xi^{\alpha-1}\right)}{\left(T^{\alpha-1}+a \xi^{\alpha-1}\right) \Gamma(\alpha)} \int_{0}^{T}(T-s)^{\alpha-1} l(s) d s+\frac{a T^{\alpha-1}}{\left(T^{\alpha-1}+a \xi^{\alpha-1}\right) \Gamma(\alpha)} \int_{0}^{\xi}(\xi-s)^{\alpha-1} l(s) d s\right] \\
= & A\|v\| \mid B .
\end{aligned}
$$


Therefore, $\lambda \leq A+\frac{B}{C}=A+\frac{B}{B(1-A)^{-1}}=A+(1-A)=1$. This contradicts $\lambda>1$. By Lemma $6, F$ has a fixed point $u^{*} \in \bar{\Omega}$. In view of $f(t, 0,0) \neq 0$, the $\operatorname{FDE}(3)$ has a nontrivial solution $u^{*} \in E$.

Now, we prove that the operator $F$ is completely continuous, we have $B_{C}=\{v \in E:\|v\| \leq C\}$ is a bounded closed convex set of $E$. We shall prove that $F\left(B_{C}\right)$ is relatively compact. The proof will be done is some steps.

(i) Let $v \in B_{C}$, we have $|F u(t)| \leq A\|v\|+B$. Consequently $F\left(B_{C}\right)$ is uniformly bounded.

(ii) Let us prove that $F\left(B_{C}\right)$ is equicontinuous. Let $t_{1}, t_{2} \in[0, T]$, with $t_{1}<t_{2}$, and $v \in B_{C}$, we have

$$
\begin{aligned}
& \left|F u\left(t_{1}\right)-F u\left(t_{2}\right)\right|=\mid \frac{1}{\Gamma(\alpha)} \int_{0}^{t_{1}}\left[\left(t_{1}-s\right)^{\alpha-1}-\frac{\left(t_{1}(T-s)\right)^{\alpha-1}}{\left(T^{\alpha-1}-a \xi^{\alpha-1}\right)}\right] f\left(s, v(s), D^{v} v(s)\right) d s \\
& -\frac{1}{\left(T^{\alpha-1}-a \xi^{\alpha-1}\right) \Gamma(\alpha)} \int_{t_{1}}^{T}\left(t_{1}(T-s)\right)^{\alpha-1} f\left(s, v(s), D^{v} v(s)\right) d s \\
& +\frac{a}{\left(T^{\alpha-1}-a \xi^{\alpha-1}\right) \Gamma(\alpha)} \int_{0}^{\xi}\left(t_{1}(\xi-s)\right)^{\alpha-1} f\left(s, v(s), D^{v} v(s)\right) d s \\
& -\frac{1}{\Gamma(\alpha)} \int_{0}^{t_{2}}\left[\left(t_{2}-s\right)^{\alpha-1}-\frac{\left(t_{2}(T-s)\right)^{\alpha-1}}{\left(T^{\alpha-1}-a \xi^{\alpha-1}\right)}\right] f\left(s, v(s), D^{v} v(s)\right) d s \\
& +\frac{1}{\left(T^{\alpha-1}-a \xi^{\alpha-1}\right) \Gamma(\alpha)} \int_{t_{2}}^{T}\left(t_{2}(T-s)\right)^{\alpha-1} f\left(s, v(s), D^{v} v(s)\right) d s \\
& -\frac{a}{\left(T^{\alpha-1}-a \xi^{\alpha-1}\right) \Gamma(\alpha)} \int_{0}^{\xi}\left(t_{2}(\xi-s)\right)^{\alpha-1} f\left(s, v(s), D^{v} v(s)\right) d s \mid \\
& =\mid \frac{1}{\Gamma(\alpha)} \int_{0}^{t_{1}}\left[\left(t_{1}-s\right)^{\alpha-1}-\frac{\left(t_{1}(T-s)\right)^{\alpha-1}}{\left(T^{\alpha-1}-a \xi^{\alpha-1}\right)}\right] f\left(s, v(s), D^{v} v(s)\right) d s \\
& -\frac{1}{\left(T^{\alpha-1}-a \xi^{\alpha-1}\right) \Gamma(\alpha)} \int_{t_{1}}^{T}\left(t_{1}(T-s)\right)^{\alpha-1} f\left(s, v(s), D^{v} v(s)\right) d s \\
& -\frac{1}{\Gamma(\alpha)} \int_{0}^{t_{2}}\left[\left(t_{2}-s\right)^{\alpha-1}-\frac{\left(t_{2}(T-s)\right)^{\alpha-1}}{\left(T^{\alpha-1}-a \xi^{\alpha-1}\right)}\right] f\left(s, v(s), D^{v} v(s)\right) d s \\
& +\frac{1}{\left(T^{\alpha-1}-a \xi^{\alpha-1}\right) \Gamma(\alpha)} \int_{t_{2}}^{T}\left(t_{2}(T-s)\right)^{\alpha-1} f\left(s, v(s), D^{v} v(s)\right) d s \\
& +\frac{a\left(t_{1}^{\alpha-1}-t_{2}^{\alpha-1}\right)}{\left(T^{\alpha-1}-a \xi^{\alpha-1}\right) \Gamma(\alpha)} \int_{0}^{\xi}(\xi-s)^{\alpha-1} f\left(s, v(s), D^{v} v(s)\right) d s \mid \\
& \leq \frac{1}{\Gamma(\alpha)} \int_{0}^{t_{1}}\left[\left(t_{1}-s\right)^{\alpha-1}+\frac{\left(t_{1}(T-s)\right)^{\alpha-1}}{\left(T^{\alpha-1}+a \xi^{\alpha-1}\right)}\right]\left|f\left(s, v(s), D^{v} v(s)\right)\right| d s \\
& +\frac{1}{\left(T^{\alpha-1}+a \xi^{\alpha-1}\right) \Gamma(\alpha)} \int_{t_{1}}^{T}\left(t_{1}(T-s)\right)^{\alpha-1}\left|f\left(s, v(s), D^{v} v(s)\right)\right| d s \\
& +\frac{1}{\Gamma(\alpha)} \int_{0}^{t_{2}}\left[\left(t_{2}-s\right)^{\alpha-1}+\frac{\left(t_{2}(T-s)\right)^{\alpha-1}}{\left(T^{\alpha-1}+a \xi^{\alpha-1}\right)}\right]\left|f\left(s, v(s), D^{v} v(s)\right)\right| d s \\
& +\frac{1}{\left(T^{\alpha-1}+a \tilde{\zeta}^{\alpha-1}\right) \Gamma(\alpha)} \int_{t_{2}}^{T}\left(t_{2}(T-s)\right)^{\alpha-1}\left|f\left(s, v(s), D^{v} v(s)\right)\right| d s \\
& +\frac{a\left|t_{1}^{\alpha-1}-t_{2}^{\alpha-1}\right|}{\left(T^{\alpha-1}-a \xi^{\alpha-1}\right) \Gamma(\alpha)} \int_{0}^{\xi}(\xi-s)^{\alpha-1}\left|f\left(s, v(s), D^{v} v(s)\right)\right| d s .
\end{aligned}
$$

Therefore,

$$
\begin{aligned}
& \left|F u\left(t_{1}\right)-F u\left(t_{2}\right)\right| \leq \frac{1}{\Gamma(\alpha)} \int_{t_{2}}^{t_{1}}\left[\left(\left(t_{1}-s\right)^{\alpha-1}-\left(t_{2}-s\right)^{\alpha-1}\right)+\frac{\left[\left(t_{1}(T-s)\right)^{\alpha-1}-\left(t_{2}(T-s)\right)^{\alpha-1}\right]}{\left(T^{\alpha-1}+a \xi^{\alpha-1}\right)}\right] \\
& \times\left|f\left(s, v(s), D^{v} v(s)\right)\right| d s+\frac{1}{\left(T^{\alpha-1}+a \xi^{\alpha-1}\right) \Gamma(\alpha)} \int_{t_{1}}^{t_{2}}\left[\left(t_{1}(T-s)\right)^{\alpha-1}-\left(t_{2}(T-s)\right)^{\alpha-1}\right] \\
& \times\left|f\left(s, v(s), D^{v} v(s)\right)\right| d s+\frac{a\left|t_{1}^{\alpha-1}-t_{2}^{\alpha-1}\right|}{\left(T^{\alpha-1}-a \xi^{\alpha-1}\right) \Gamma(\alpha)} \int_{0}^{\tau}(\xi-s)^{\alpha-1}\left|f\left(s, v(s), D^{v} v(s)\right)\right| d s .
\end{aligned}
$$


Letting $t_{1} \rightarrow t_{2}$, then $\left|F u\left(t_{1}\right)-F u\left(t_{2}\right)\right|$ tends to 0 . Consequently $F\left(B_{C}\right)$ is equicontinuous. From Ascoli-Arzela theorem, we deduce that $F$ is a completely continuous. This completes the proof.

Theorem 2. Suppose that $f(t, 0,0) \neq 0, T^{\alpha-1}+a \xi^{\alpha-1} \neq 0$, and there exist nonnegative functions $k, h, l \in L^{1}[0, T]$ such that $|f(t, x, y)| \leq k(t)|x|+h(t)|y|+l(t)$, a.e. $(t, x, y) \in[0, T] \times \mathbf{R}^{2}$. If one of the following conditions is fulfilled;

(1) There exists a constant $p>1$ such that

$$
\int_{0}^{1}(k(s)+h(s))^{p} d s<\left[\frac{\left(T^{\alpha-1}+a \xi^{\alpha-1}\right) \Gamma(\alpha)(1+q(\alpha-1))^{1 / q}}{\left(2 T^{\alpha-1}+a \xi^{\alpha-1}\right) T^{(1+q(\alpha-1)) / q}+a T^{\alpha-1} \xi^{(1+q(\alpha-1)) / q}}\right]^{p}, \frac{1}{p}+\frac{1}{q}=1,
$$

(2) $k(s)+h(s)$ satisfies

$$
\begin{gathered}
k(s)+h(s) \leq \frac{\alpha \Gamma(\alpha)\left(T^{\alpha-1}+a \xi^{\alpha-1}\right)}{\left(2 T^{\alpha-1}+a \xi^{\alpha-1}\right) T^{\alpha}+a T^{\alpha-1} \xi^{\alpha}}, \quad \text { a.e. } s \in[0, T], \\
\operatorname{meas}\left\{s \in[0, T]: k(s)+h(s)<\frac{\alpha \Gamma(\alpha)\left(T^{\alpha-1}+a \xi^{\alpha-1}\right)}{\left(2 T^{\alpha-1}+a \xi^{\alpha-1}\right) T^{\alpha}+a T^{\alpha-1} \xi^{\alpha}}\right\}>0 .
\end{gathered}
$$

Then the FDE (3) has at least one nontrivial solution $u^{*} \in E$.

Proof. Let $A$ be defined as in the proof of Theorem 1 . To prove Theorem 2, we only need to prove that $A<1$. Since $T^{\alpha-1}+a \xi^{\alpha-1} \neq 0$, we have

$$
A=\frac{2 T^{\alpha-1}+a \xi^{\alpha-1}}{\left(T^{\alpha-1}+a \xi^{\alpha-1}\right) \Gamma(\alpha)} \int_{0}^{T}(T-s)^{\alpha-1}(k(s)+h(s)) d s+\frac{a T^{\alpha-1}}{\left(T^{\alpha-1}+a \xi^{\alpha-1}\right) \Gamma(\alpha)} \int_{0}^{\xi}(\xi-s)^{\alpha-1}(k(s)+h(s)) d s .
$$

(1) Using the Hölder inequality, we have

$$
\begin{aligned}
A \leq & {\left[\int_{0}^{1}(k(s)+h(s))^{p} d s\right]^{1 / p}\left\{\frac{2 T^{\alpha-1}+a \xi^{\alpha-1}}{\left(T^{\alpha-1}+a \xi^{\alpha-1}\right) \Gamma(\alpha)}\left[\int_{0}^{T}\left((T-s)^{\alpha-1}\right)^{q} d s\right]^{1 / q}\right.} \\
& \left.+\frac{a T^{\alpha-1}}{\left(T^{\alpha-1}+a \xi^{\alpha-1}\right) \Gamma(\alpha)}\left[\int_{0}^{\xi}\left((\xi-s)^{\alpha-1}\right)^{q} d s\right]^{1 / q}\right\} \\
\leq & {\left[\int_{0}^{1}(k(s)+h(s))^{p} d s\right]^{1 / p}\left\{\frac{2 T^{\alpha-1}+a \xi^{\alpha-1}}{\left(T^{\alpha-1}+a \xi^{\alpha-1}\right) \Gamma(\alpha)}\left[\frac{T^{1+q(\alpha-1)}}{(1+q(\alpha-1))}\right]^{1 / q}\right.} \\
& \left.+\frac{a T^{\alpha-1}}{\left(T^{\alpha-1}+a \xi^{\alpha-1}\right) \Gamma(\alpha)}\left[\frac{\xi^{1+q(\alpha-1)}}{1+q(\alpha-1)}\right]^{1 / q}\right\} \\
\leq & {\left[\int_{0}^{1}(k(s)+h(s))^{p} d s\right]^{1 / p}\left[\frac{\left(2 T^{\alpha-1}+a \xi^{\alpha-1}\right) T^{(1+q(\alpha-1)) / q}}{\left(T^{\alpha-1}+a \xi^{\alpha-1}\right) \Gamma(\alpha)(1+q(\alpha-1))^{1 / q}}+\frac{a T^{\alpha-1} \xi^{(1+q(\alpha-1)) / q}}{\left(T^{\alpha-1}+a \xi^{\alpha-1}\right) \Gamma(\alpha)(1+q(\alpha-1))^{1 / q}}\right] } \\
\leq & \frac{\left(T^{\alpha-1}+a \xi^{\alpha-1}\right) \Gamma(\alpha)(1+q(\alpha-1))^{1 / q}}{\left(2 T^{\alpha-1}+a \xi^{\alpha-1}\right) T^{(1+q(\alpha-1)) / q}+a T^{\alpha-1} \xi^{(1+q(\alpha-1)) / q}} \times \frac{\left(2 T^{\alpha-1}+a \xi^{\alpha-1}\right) T^{(1+q(\alpha-1)) / q}+a T^{\alpha-1} \xi^{(1+q(\alpha-1)) / q}}{\left(T^{\alpha-1}+a \xi^{\alpha-1}\right) \Gamma(\alpha)(1+q(\alpha-1))^{1 / q}} \\
= & 1 .
\end{aligned}
$$

(2) In this case, we have

$$
\begin{aligned}
A \leq & \frac{\alpha \Gamma(\alpha)\left(T^{\alpha-1}+a \xi^{\alpha-1}\right)}{\left(2 T^{\alpha-1}+a \xi^{\alpha-1}\right) T^{\alpha}+a T^{\alpha-1} \xi^{\alpha}}\left[\frac{2 T^{\alpha-1}+a \xi^{\alpha-1}}{\left(T^{\alpha-1}+a \xi^{\alpha-1}\right) \Gamma(\alpha)} \int_{0}^{T}(T-s)^{\alpha-1} d s\right. \\
& \left.+\frac{a T^{\alpha-1}}{\left(T^{\alpha-1}+a \xi^{\alpha-1}\right) \Gamma(\alpha)} \int_{0}^{\xi}(\xi-s)^{\alpha-1} d s\right]
\end{aligned}
$$




$$
\begin{aligned}
& \leq \frac{\alpha \Gamma(\alpha)\left(T^{\alpha-1}+a \xi^{\alpha-1}\right)}{\left(2 T^{\alpha-1}+a \xi^{\alpha-1}\right) T^{\alpha}+a T^{\alpha-1} \xi^{\alpha}}\left[\frac{\left(2 T^{\alpha-1}+a \xi^{\alpha-1}\right) T^{\alpha}}{\alpha \Gamma(\alpha)\left(T^{\alpha-1}+a \xi^{\alpha-1}\right)}+\frac{a T^{\alpha-1} \xi^{\alpha}}{\alpha \Gamma(\alpha)\left(T^{\alpha-1}+a \xi^{\alpha-1}\right)}\right] \\
& \leq \frac{\alpha \Gamma(\alpha)\left(T^{\alpha-1}+a \xi^{\alpha-1}\right)}{\left(2 T^{\alpha-1}+a \xi^{\alpha-1}\right) T^{\alpha}+a T^{\alpha-1} \xi^{\alpha}} \cdot \frac{\left(2 T^{\alpha-1}+a \xi^{\alpha-1}\right) T^{\alpha}+a T^{\alpha-1} \xi^{\alpha}}{\alpha \Gamma(\alpha)\left(T^{\alpha-1}+a \xi^{\alpha-1}\right)}=1 .
\end{aligned}
$$

This completes the proof.

Corollary 1. Suppose $f(t, 0,0) \neq 0,(1+a) T^{\alpha-1} \neq 0$, and there exist nonnegative functions $k, h, l \in L^{1}[0, T]$ such that $|f(t, x, y)| \leq k(t)|x|+h(t)|y|+l(t)$, a.e. $(t, x, y) \in[0, T] \times \mathbf{R}^{2}$. If one of following conditions is fulfilled;

(1) There exists a constant $p>1$ such that

$$
\int_{0}^{1}(k(s)+h(s))^{p} d s<\left[\frac{(1+a) T^{\alpha-1} \Gamma(\alpha)(1+q(\alpha-1))^{1 / q}}{2(1+a) T^{\alpha-1} T^{(1+q(\alpha-1)) / q}}\right]^{p}, \frac{1}{p}+\frac{1}{q}=1 .
$$

(2) $k(s)+h(s)$ satisfies

$$
\begin{gathered}
k(s)+h(s) \leq \frac{\alpha \Gamma(\alpha)}{2 T^{\alpha}}, \quad \text { a.e. } s \in[0, T], \\
\text { meas }\left\{s \in[0, T]: k(s)+h(s)<\frac{\alpha \Gamma(\alpha)}{2 T^{\alpha}}\right\}>0 .
\end{gathered}
$$

Then, the FDE (3) has at least one nontrivial solution $u^{*} \in E$.

Proof. In this case, we have

$$
\begin{aligned}
A & =\frac{2 T^{\alpha-1}+a \xi^{\alpha-1}}{\left(T^{\alpha-1}+a \xi^{\alpha-1}\right) \Gamma(\alpha)} \int_{0}^{T}(T-s)^{\alpha-1}(k(s)+h(s)) d s+\frac{a T^{\alpha-1}}{\left(T^{\alpha-1}+a \xi^{\alpha-1}\right) \Gamma(\alpha)} \int_{0}^{\xi}(\xi-s)^{\alpha-1}(k(s)+h(s)) d s \\
& \leq \frac{2 T^{\alpha-1}+a T^{\alpha-1}}{\left(T^{\alpha-1}+a T^{\alpha-1}\right) \Gamma(\alpha)} \int_{0}^{T}(T-s)^{\alpha-1}(k(s)+h(s)) d s+\frac{a T^{\alpha-1}}{\left(T^{\alpha-1}+a T^{\alpha-1}\right) \Gamma(\alpha)} \int_{0}^{T}(T-s)^{\alpha-1}(k(s)+h(s)) d s \\
& =\frac{2(1+a) T^{\alpha-1}}{(1+a) T^{\alpha-1} \Gamma(\alpha)} \int_{0}^{T}(T-s)^{\alpha-1}(k(s)+h(s)) d s .
\end{aligned}
$$

Proof of this Corollary 1 is similar to the proof Theorem 2. This completes the proof.

\section{Applications}

In order to illustrate the above results, we consider some examples.

Example 1. Consider the following system of FDE

$$
\left\{\begin{array}{l}
D^{3 / 2} u(t)=\frac{t}{207} v(t)+\frac{t+2}{100} D^{5 / 4} v(t)+t^{2}-1, \quad t \in(0, T) \\
u(0)=0, \quad u(T)=2 u(T / 2)
\end{array}\right.
$$

Set $\alpha=3 / 2, a=2, \xi=T / 2$, and

$$
\begin{aligned}
& f(t, x, y)=\frac{t}{207} x(t)+\frac{t+2}{100} y(t)+t^{2}-1, \\
& k(t)=\frac{t}{100}, \quad h(t)=\frac{t+2}{100}, \quad l(t)=t^{2} .
\end{aligned}
$$

It is easy to prove that $k, h, l \in L^{1}[0, T]$ are nonnegative functions, and

$$
|f(t, x, y)| \leq k(t)|x|+h(t)|y|+l(t), \quad \text { a.e. }(t, x, y) \in[0, T] \times \mathbf{R}^{2}
$$

and

$$
T^{\alpha-1}+a \xi^{\alpha-1}=\left(1+\frac{2}{2^{1 / 2}}\right) T^{1 / 2} \neq 0
$$


Moreover, we have

$$
\begin{gathered}
A=\frac{2 T^{\alpha-1}+a \xi^{\alpha-1}}{\left(T^{\alpha-1}+a \xi^{\alpha-1}\right) \Gamma(\alpha)} \int_{0}^{T}(T-s)^{\alpha-1}(k(s)+h(s)) d s+\frac{a T^{\alpha-1}}{\left(T^{\alpha-1}+a \xi^{\alpha-1}\right) \Gamma(\alpha)} \int_{0}^{\xi}(\xi-s)^{\alpha-1}(k(s)+h(s)) d s, \\
A \approx 13 \cdot 10^{-3} \cdot T^{3 / 2}+4 \cdot 10^{-3} \cdot T^{5 / 2}<1 .
\end{gathered}
$$

Hence, by Theorem 1, the FDE (4) has at least one nontrivial solution $u^{*}$ in $E$.

Example 2. Consider the following system of FDE

$$
\left\{\begin{array}{l}
D^{1 / 2} u(t)=\frac{\sqrt[3]{1+t^{5}}}{20} v(t) \sin v(t)+\frac{\sqrt[3]{1+t^{5}}}{5} D^{3 / 4} v(t)+\cos t-e^{t}, \quad t \in(0, T) \\
u(0)=0, \quad u(T)=4 u(T / 3) .
\end{array}\right.
$$

Set $\alpha=1 / 2, a=4, \xi=T / 3$, and

$$
\begin{aligned}
& f(t, x, y)=\frac{\sqrt[3]{1+t^{5}}}{20} x(t) \sin x(t)+\frac{\sqrt[3]{1+t^{5}}}{5} y(t)+\cos t-e^{t}, \\
& k(t)=\sqrt[3]{1+t^{5}} / 10, \quad h(t)=\sqrt[3]{1+t^{5}} / 4, \quad l(t)=\cos t+e^{t} .
\end{aligned}
$$

It is easy to prove that $k, h, l \in L^{1}[0, T]$ are nonnegative functions, and

$$
|f(t, x, y)| \leq k(t)|x|+h(t)|y|+l(t), \quad \text { a.e. }(t, x, y) \in[0, T] \times \mathbf{R}^{2},
$$

and

$$
T^{\alpha-1}+a \xi^{\alpha-1}=\left(1+\frac{4}{3^{-1 / 2}}\right) T^{-1 / 2} \neq 0 .
$$

Let $p=3, q=3 / 2$, such that $\frac{1}{p}+\frac{1}{q}=1$, then

$$
\int_{0}^{1}(k(s)+h(s))^{p} d s=\frac{2401}{48000} \approx 0.05 .
$$

Moreover, we have

$$
\left[\frac{\left(T^{\alpha-1}+a \xi^{\alpha-1}\right) \Gamma(\alpha)(1+q(\alpha-1))^{1 / q}}{\left(2 T^{\alpha-1}+a \xi^{\alpha-1}\right) T^{(1+q(\alpha-1)) / q}+a T^{\alpha-1} \xi^{(1+q(\alpha-1)) / q}}\right]^{p} \approx 0.51 . T^{-1 / 2} .
$$

Therefore,

$$
\int_{0}^{1}(k(s)+h(s))^{p} d s<\left[\frac{\left(T^{\alpha-1}+a \xi^{\alpha-1}\right) \Gamma(\alpha)(1+q(\alpha-1))^{1 / q}}{\left(2 T^{\alpha-1}+a \xi^{\alpha-1}\right) T^{(1+q(\alpha-1)) / q}+a T^{\alpha-1} \xi^{(1+q(\alpha-1)) / q}}\right]^{p} .
$$

Hence, by Theorem 2(1), the FDE (5) has at least one nontrivial solution $u^{*}$ in $E$.

Example 3. Consider the following system of FDE

$$
\left\{\begin{array}{l}
D^{3 / 2} u(t)=\frac{\sqrt{t}}{2\left(\frac{1}{2}+v(t)\right)} e^{\left|v^{2}(t)-1\right|} \cos v(t)+\frac{\left(1+t^{2}\right)}{9+e^{t}} D^{7 / 3} v(t)+e^{-t}-\sin t, \quad t \in(0, T) \\
u(0)=0, \quad u(T)=3 u(T / 4) .
\end{array}\right.
$$

Set $\alpha=3 / 2, a=3, \xi=T / 4$, and

$$
f(t, x, y)=\frac{\sqrt{t}}{2\left(\frac{1}{2}+x(t)\right)} e^{\left|x^{2}(t)-1\right|} \cos x(t)+\frac{\left(1+t^{2}\right)}{9+e^{t}} y(t)+e^{-t}-\sin t,
$$




$$
k(t)=\frac{\sqrt{t}}{2}, \quad h(t)=\frac{\left(1+t^{2}\right)}{3}, \quad l(t)=e^{-t}+\sin t .
$$

It is easy to prove that $k, h, l \in L^{1}[0, T]$ are nonnegative functions, and

$$
|f(t, x, y)| \leq k(t)|x|+h(t)|y|+l(t), \quad \text { a.e. }(t, x) \in[0, T] \times \mathbf{R}^{2},
$$

and

$$
T^{\alpha-1}+a \xi^{\alpha-1}=\left(1+\frac{3}{4^{1 / 2}}\right) T^{1 / 2} \neq 0 .
$$

Moreover, we have

$$
\frac{\alpha \Gamma(\alpha)\left(T^{\alpha-1}+a \xi^{\alpha-1}\right)}{\left(2 T^{\alpha-1}+a \xi^{\alpha-1}\right) T^{\alpha}+a T^{\alpha-1} \xi^{\alpha}}=\frac{15 \sqrt{\pi}}{31} T^{-3 / 2} .
$$

Therefore,

$$
\begin{gathered}
k(s)+h(s)=\frac{\sqrt{s}}{2}+\frac{\left(1+s^{2}\right)}{3}<\frac{15 \sqrt{\pi}}{31} T^{-3 / 2}, \quad s \in[0, T], \\
\text { meas }\left\{s \in[0, T]: k(s)+h(s)<\frac{\alpha \Gamma(\alpha)\left(T^{\alpha-1}+a \xi^{\alpha-1}\right)}{\left(2 T^{\alpha-1}+a \xi^{\alpha-1}\right) T^{\alpha}+a T^{\alpha-1} \xi^{\alpha}}\right\}>0 .
\end{gathered}
$$

Hence, by Theorem 2(2), the FDE (6) has at least one nontrivial solution $u^{*}$ in $E$.

Acknowledgments: The authors want to thank the anonymous referee for the throughout reading of the manuscript and several suggestions that help us improve the presentation of the paper.

Author Contributions: All authors contributed equally to the writing of this paper. All authors read and approved the final manuscript.

Conflicts of Interest: "The authors declare no conflict of interest."

\section{References}

[1] Cuesta, E., \& Finat, J. (2003). Image processing by means of a linear integro-differential equation. In $3 r d$ IASTED International Conference Visualization, Imaging and Image Processing, 1 , 438-442.

[2] Deng, W. (2007). Short memory principle and a predictorÜcorrector approach for fractional differential equations. Journal of Computational and Applied Mathematics, 206(1), 174-188.

[3] Kilbas, A. A., Srivastava, H. M., \& Trujillo, J. J. (2006). Theory and Applications of Fractional Differential Equations (Vol. 204). elsevier.

[4] Lakshmikantham, V., \& Vatsala, A. S. (2008). Basic theory of fractional differential equations. Nonlinear Analysis: Theory, Methods \& Applications, 69(8), 2677-2682.

[5] Lakshmikantham, V., Leela, S., \& Devi, J. V. (2009). Theory of Fractional Dynamic Systems. Cambridge Academic, Cambridge, UK.

[6] Metzler, R., Schick, W., Kilian, H. G., \& Nonnenmacher, T. F. (1995). Relaxation in filled polymers: A fractional calculus approach. The Journal of Chemical Physics, 103(16), 7180-7186.

[7] Miller, K. S., \& Ross, B. (1993). An Introduction to the Fractional Calculus and Fractional Differential Equations. John Wiley and Sons, Inc. New York.

[8] Podlubny, I. (1994). Fractional-order systems and fractional-order controllers. Institute of Experimental Physics, Slovak Academy of Sciences, Kosice, 12(3), 1-18.

[9] Podlubny, I. (1999). Fractional Diffrential Equations, Academic Press, San Diego.

[10] Ahmad, B., \& Nieto, J. J. (2009). Existence results for a coupled system of nonlinear fractional differential equations with three-point boundary conditions. Computers $\mathcal{E}$ Mathematics with Applications, 58(9), 1838-1843.

[11] Aghajani, A., Pourhadi, E., \& Trujillo, J. J. (2013). Application of measure of noncompactness to a Cauchy problem for fractional differential equations in Banach spaces. Fractional Calculus and Applied Analysis, 16(4), 962-977.

[12] Jleli, M., Mursaleen, M., \& Samet, B. (2016). Q-Integral equations of fractional orders. Electronic Journal of Differential Equations, 2016(17), 1-14.

[13] Li, K., \& Peng, J., \& Gao, J. (2013). Nonlocal Fractional Differential Equations in Separable Banach Spaces, Electronic Journal of Differential Equations, 2013(7), 1-7.

[14] Persidskii, K. P. (1959). Countable systems of differential equations and stability of their solutions. Izvestiya Akademii Nauk Kazakh SSR, 7, 52-71. 
[15] Voigt, A. (1974). Line method approximations to the Cauchy problem for nonlinear parabolic differential equations. Numerische Mathematik, 23(1), 23-36.

[16] Deimling, K. (1977). Ordinary Differential Equations in Banach Spaces, Lect. Notes. Math. 596, Springer, Berlin.

[17] Zautokov, O. A. (1965). Countable system of differential equations and their applications, Diff. Uravn, 1, $162-170$.

[18] Mursaleen, M., Bilalov, B., \& Rizvi, S. M. (2017). Applications of measures of noncompactness to infinite system of fractional differential equations. Filomat, 31(11), 3421-3432.

[19] Deimling, K. (1985). Nonlinear Functional Analysis, Springer, Berlin.

[20] Diethelm, K. (2010). The Analysis of Fractional Differential Equations, Springer-Verlag Berlin Heidelberg .

[21] Goodrich, C., \& Peterson, A. C. (2015). Discrete Fractional Calculus, Springer International Publishing Switzerland .

[22] Herrmann, R. (2014). Fractional Calculus an Tntroduction for Physicists, GigaHedron, Germany .

[23] Rajkovic, P., Marinkovic, S., \& Stankovic, M. (2007). On qÜAnalogues of Caputo Derivative and MittagÜLeffler Function. Fractional calculus and applied analysis, 10(4), 359-373.

(C) 2020 by the authors; licensee PSRP, Lahore, Pakistan. This article is an open access article distributed under the terms and conditions of the Creative Commons Attribution (CC-BY) license (http://creativecommons.org/licenses/by/4.0/). 